\title{
Estabilidad oxidativa del aceite de hígado de merluza
}

\author{
Por A. Rodríguez (1), D. Barrera-Arellano (2) y M.A. Grompone *(1) \\ 1. Universidad de la República. Facultad de Química. Casilla de Correos 1157. Montevideo. Uruguay. \\ 2. Lab. Oleos e Gorduras. FEA/UNICAMP. Caixa Postal 6091. Campinas. SP 13081-970 Brasil.
}

\section{RESUMEN}

\section{Estabilidad oxidativa del aceite de hígado de merluza}

Se estudió la estabilidad oxidativa de aceite de hígado de merluza industrial y el mejoramiento de la misma mediante el empleo de varios antioxidantes. Para ello se determinó el periodo de inducción (horas) en el Rancimat $\left(70^{\circ} \mathrm{C}\right.$, flujo de aire $8,3 \mathrm{l} / \mathrm{h}$ y 5 gramos de muestra). Los antioxidantes usados fueron BHT, BHA, TBHQ, palmitato de ascorbilo (PA) y galato de propilo (GP) y los preparados comerciales Ronoxan A, Tocomix D y Antracine 220.

El antioxidante más eficiente fue TBHQ en la concentración de $0.02 \%$ (aumentando el periodo de inducción 6,5 veces respecto al aceite sin antioxidantes). Los antioxidantes naturales no mejoraron significativamente la estabilidad oxidativa del aceite, utilizados en la concentración de $0,02 \%$.

PALABRAS-CLAVE: Aceite - Antioxidante (efecto) - Estabilidad oxidativa - Higado (merluza)

\section{SUMMARY}

Oxidative stability of hake liver oil.

Oxidative stability of industrial hake liver oil and its improvement by means of antioxidants were studied. For this purpose the induction period (hours) was determined in a Rancimat $\left(70^{\circ} \mathrm{C}\right.$, air flow $8.3 \mathrm{l} / \mathrm{h}$ and $5 \mathrm{~g}$ sample). The antioxidants employed were $\mathrm{BHT}$, BHA, TBHQ, ascorbyl palmitate and propyl gallate and the commercial products Ronoxan A, Tocomix D and Antracine 220

The most efficient antioxidant was TBHQ $0,02 \%$ (the induction period was increased 6,5 times with regard to the oil without antioxidant). The oxidative stability of the oil did not improve significatively with $0,02 \%$ of natural antioxidants. lity.

KEY-WORDS: Antioxidant (effect) - Liver (hake) - Oil - Oxidative stabi-

\section{INTRODUCCION}

En Uruguay, uno de los recursos pesqueros más explotados es la merluza común (Merluccius hubbsi), que pertenece a la familia de los Merlúccidos (orden Gadiformes) y se distribuye en el océano Atlántico sud-occidental. La industria del fileteado de merluza es de gran importancia, y los productos de desecho (los hígados, por ejemplo) son generalmente sub-aprovechados. El hígado de merluza contiene entre 35 y $50 \%$ de aceite en peso, por lo que constituye una valiosa fuente de ácidos grasos poliinsaturados de la serie $w-3$, típicos de los aceites de pescado.

El creciente interés en aumentar la ingesta de ácidos grasos poliinsaturados ha hecho que se intenten desarrollar nuevos alimentos o complementos alimenticios con aceites de pescado en su composición. Estos aceites son muy sensibles a la oxidación, debido a su alto porcentaje de ácidos grasos poliinsaturados. Estudios recientes han sugerido que los aceites de pescado no estabilizados podrían significar la ingestión de compuestos potencialmente tóxicos producidos durante la oxidación de los ácidos grasos poliinsaturados de la serie $w-3$ (Shukla, 1991).

Para retardar el deterioro de los aceites altamente insaturados se han utilizado antioxidantes sintéticos y naturales. Entre los sintéticos, la tert-butilhidroxiquinona (TBHQ) es considerada sensiblemente más eficiente que el butilhidroxianisol (BHA) y que el butilhidroxitolueno (BHT) (Ke, 1977; Pacheco, 1991 a; Kaitaranta, 1992). Algunos antioxidantes naturales, como los tocoferoles, son citados como efectivos si se utilizan en altas concentraciones (Ke, 1977), y también sus combinaciones con extractos de romero (Wada, 1992) y con ácido ascórbico (Han, 1991). Thorisson et al. (1992) y Koning-Milkovitch (1991) estudiaron las propiedades antioxidantes de la etoxiquina sobre los aceites de pescado y Lovaas (1991) estudió el efecto de las poliaminas. Ninguno de estos trabajos menciona la estabilización del aceite de hígado de merluza.

La oxidación de un aceite ocurre en dos etapas: un periodo de inducción en el que ésta tiene lugar lentamente y a una velocidad relativamente uniforme, y una segunda etapa en la que hay un aumento rápido de la oxidación, iniciándose las señales perceptibles de sabor y olor características de la rancidez. La duración del periodo de inducción se puede tomar como una medida de su resistencia a la oxidación (Sherwin, 1968). El método Rancimat es una versión modificada y automatizada del Método del Oxígeno Activo (AOM), método oficial Cd 1257 (AOCS, 1988) (de Man, 1984; Läubli, 1986). De acuerdo con el mecanismo propuesto (de Man 1987), 
al final del periodo de inducción, los radicales libres alfahidroperoxi aldehído, por rotura de enlaces -C-C y -O-O, dan lugar a la formación de ácido fórmico. Si el aire emergente del aceite se hace burbujear en agua desionizada, los ácidos volátiles se pueden determinar conductimétricamente.

El presente trabajo estudia el mejoramiento de la estabilidad oxidativa del aceite de hígado de merluza por el agregado de antioxidantes, mediante la determinación del periodo de inducción $(\mathrm{PI})$ según el método Rancimat.

\section{MATERIALES Y METODOS}

\subsection{Materiales.}

Se utilizó aceite de hígado de merluza crudo producido industrialmente en Uruguay. Este aceite fue extraído en agosto de 1992 y conservado en recipientes bien cerrados sin aire a temperatura ambiente $\left(20^{\circ} \mathrm{C}\right)$.

Los antioxidantes usados fueron BHA (Fluka), BHT (Fluka), TBHQ (Fluka), galato de propilo (Fluka), palmitato de ascorbilo (Merck) y los siguientes preparados comerciales:

Tocomix D (J. Dekker): gama y alfa tocoferoles $60 \%$ + citrato de monoglicéridos.

Antracine 220 (J. Dekker): TBHQ 40\% + ácido cítrico + propilenglicol.

Ronoxan A (Roche): $\quad$ PA $25 \%$ + lecitina $70 \%$ + alfa tocoferol $5 \%$.

Se prepararon soluciones al $1 \%$ de los antioxidantes en aceite de hígado de merluza o en propilenglicol. Luego se añadieron directamente a las muestras las cantidades adecuadas de dichas soluciones concentradas.

\subsection{Métodos.}

a) Pretratamiento de la muestra.

La muestra de aceite crudo se secó con sulfato de sodio anhidro y se filtró, conservándose posteriormente a $4^{\circ} \mathrm{C}$.

\section{b) Caracterización y composición del aceite.}

Fueron determinados en el aceite, en ensayos por duplicado:

-índice de acidez

- índice de peróxido

Método Ca 5a-40 (AOCS)

- sustancias polares

Método Cd 8-53 (AOCS)

Método 2.507 (IUPAC)

La composición en ácidos grasos se determinó por cromatografía de gases de los ésteres metílicos obtenidos según Hartman \& Lago (1973). Se utilizó una columna de acero inoxidable (4 m por 1/8") empacada con $10 \%$ Silar $10 \mathrm{C}$. El análisis fue realizado en un cromatógrafo Perkin Elmer Sigma 3B con detector de ioniza- ción de llama e integrador Perkin Elmer LCI-100. La temperatura de la columna fue de $175^{\circ} \mathrm{C}$, la del detector e inyector de $225^{\circ} \mathrm{C}$ y el flujo de nitrógeno de $25 \mathrm{ml} / \mathrm{min}$. La identificación fue hecha por comparación de los tiempos de retención con patrones y gráficas de tiempo de retención relativo vs. $n^{\circ}$ de carbonos (Ackman, 1969). Según el método oficial de la American Oil Chemists Society Ce 1-62 (AOCS, 1988) la reproducibilidad del método es de un $10 \%$ relativo en los picos mayores de $5 \%$ y ésta disminuye gradualmente en los picos menores de $5 \%$.

Para la determinación de la estabilidad oxidativa se utilizó un equipo Rancimat 617 Metrohm, con 5 gramos de muestra, flujo de aire $8,3 \mathrm{l} / \mathrm{h}$ y temperatura $70^{\circ} \mathrm{C}$. Cada ensayo se realizó por duplicado.

La AOCS (Método Cd 12-57) así como de Man (1984) hacen recomendaciones muy estrictas sobre la limpieza del material de vidrio a emplear. El propio fabricante del equipo sugiere sustituir la limpieza con mezcla sulfocrómica por detergente alcalino Extran, ya que entre otras ventajas, se eliminan así los iones cromo residuales. La técnica utilizada en la limpieza del material de vidrio se basó en las especificaciones de Pacheco (1991 a), con algunas modificaciones. El material usado en contacto directo con el aceite se lavó y se trató con una solución de $\mathrm{KOH}$ alcohólica al $6 \%$ durante una hora. Luego se dejó 3 horas en una solución de detergente Extran MA 01 alcalino (Merck) al 10\%. Finalmente, se enjuagó con agua y luego con agua destilada. El material utilizado para la recolección de volátiles, se limpió con detergente Extran durante una hora y media.

\section{RESULTADOS Y DISCUSION}

La muestra analizada corresponde a una tachada de producción industrial, según sugerencia de Stansby (1991). Este autor postula que la mayoría de los investigadores que trabajan con aceites de animales marinos emplean métodos analíticos precisos pero sus resultados son de poca significación, porque se refieren a unos pocos especímenes. La composición en ácidos grasos de un aceite de pescado depende, en gran medida, del contenido lipídico del alimento con que se nutrió el pez. Dicho alimento varía según la ubicación geográfica, la época del año, las modificaciones climáticas, etc. Por eso, se sugiere estudiar muestras correspondientes a tachadas industriales para caracterizar las propiedades del aceite de una especie dada, ya que éstas corresponden a un gran número de ejemplares.

La composición en ácidos grasos del aceite se muestra en la tabla I. Considerando los ácidos grasos mayoritarios, tiene un contenido en saturados del $22,1 \%$, en monoenos de $37,4 \%$ y en poliinsaturados $32,4 \%$. Para comparación, se presenta en la tabla II la composición en ácidos grasos de los aceites de hígado de varias especies de peces.

Las características del aceite de hígado de merluza son: 
Indice de acidez: $\quad 1,4 \mathrm{mg} \mathrm{KOH} / \mathrm{g}$

Indice de peróxidos: 4,2 meq $\mathrm{O}_{2} / \mathrm{Kg}$

Sustancias polares: $4,5 \%$.

Tabla I

Composición de aceite de hígado de merluza

\begin{tabular}{cc}
\hline Acido graso & $\begin{array}{c}\text { Aceite de hígado de merluza } \\
\text { (Merluccius hubbsi) (\%) }\end{array}$ \\
\hline $14: 0$ & 4,8 \\
$16: 0$ & 15,6 \\
$16: 1$ & 6,2 \\
$18: 0$ & 1,7 \\
$18: 1$ & 16,5 \\
$18: 2$ & 2,3 \\
$18: 3+20: 1$ & 6,4 \\
$18: 4$ & 3,6 \\
$20: 4$ & 1,1 \\
$20: 5$ & 6,4 \\
$22: 1$ & 8,3 \\
$22: 5$ & 1,1 \\
$22: 6$ & 17,9 \\
No ident. & 3,6 \\
\hline Saturados & 22,1 \\
Monoinsaturados & 37,4 \\
Poliinsaturados & 32,4 \\
\hline
\end{tabular}

Tabla II

Composición de aceite de hígado de algunos peces

\begin{tabular}{cccc}
\hline Acido graso & $\begin{array}{c}\text { Caballa } \\
(\text { Scomber } \\
\text { scombrus)(\%)(1) }\end{array}$ & $\begin{array}{c}\text { Bacalao } \\
\text { Gadus morhua) } \\
(\%)(2)\end{array}$ & $\begin{array}{c}\text { Tiburón } \\
(\text { Prionace } \\
\text { glauca)(\%)(3) }\end{array}$ \\
\hline $14: 0$ & 2,1 & 4,2 & 4,2 \\
$16: 0$ & 17,4 & 10,6 & 19,8 \\
$16: 1$ & 6,2 & 9,5 & 7,7 \\
$18: 0$ & 2,2 & 1,4 & 3,8 \\
$18: 1$ & 31,6 & 21,1 & 24,8 \\
$18: 2$ & 1,9 & 1,0 & 0,6 \\
$18: 3$ & - & 0,4 & - \\
$20: 1$ & 4,2 & 19,0 & 7,8 \\
$18: 4$ & 0,7 & 0,8 & - \\
$20: 4$ & - & 0,4 & 2,9 \\
$20: 5$ & 7,9 & 9,5 & 4,6 \\
$22: 1$ & 1,8 & 7,3 & - \\
$22: 5$ & - & 0,6 & 1,7 \\
$22: 6$ & 12,5 & 12,6 & 18,9 \\
\hline Saturados & 21,7 & 16,2 & 27,8 \\
Monoinsaturados & 43,8 & 56,9 & 40,3 \\
Poliinsaturados & 23,0 & 25,3 & 28,7 \\
\hline
\end{tabular}

(1) Gerbshbein, 1969

(2) Hardy, 1972

(3) Pacheco, $1991 \mathrm{~b}$

La Ordenanza Bromatológica de Montevideo indica que los límites aceptables para un aceite comestible son de un $0,3 \%$ de acidez expresada en ácido oleico y de 10 meq $\mathrm{O}_{2} / \mathrm{Kg}$ de índice de peróxido (Digesto Municipal, 1990). En España, es admitido hasta un $25 \%$ de com- puestos polares en aceites para frituras (España, 1989) Los bajos valores de estos índices en el aceite indican su aptitud para realizar los ensayos, ya que su deterioro oxidativo es mínimo al comienzo del trabajo.

Los efectos inhibitorios de varios antioxidantes sobre el aceite de hígado de merluza, determinados por el método Rancimat, se muestran en la tabla III. En la concentración de $0,02 \%$ de antioxidante el orden de eficiencia fue $\mathrm{TBHQ}>\mathrm{GP}>\mathrm{BHA}>\mathrm{BHT}>\mathrm{PA}>$ tocoferoles. TBHQ aumenta 6,5 veces el periodo de inducción del aceite y el siguiente en la escala, GP, sólo 2,6 veces.

Tabla III

Efecto de los antioxidantes en la estabilidad oxidativa del aceite de hígado de merluza

\begin{tabular}{|c|c|c|c|}
\hline \multicolumn{2}{|c|}{ Muestra } & $\begin{array}{l}\text { Periodo de } \\
\text { inducción } \\
\text { (horas) }\left(^{*}\right)\end{array}$ & $\begin{array}{l}\text { Eficiencia del } \\
\text { antioxidante }\end{array}$ \\
\hline \multicolumn{2}{|c|}{ Aceite } & 5,4 & 1,0 \\
\hline “ & + Tocomix D $(0,034 \%)$ & 5,5 & 1,0 \\
\hline “ & $+\mathrm{PA}(0,02 \%)$ & 6,2 & 1,1 \\
\hline “ & $+\mathrm{PA}(0,05 \%)$ & 6,1 & 1,1 \\
\hline “ & + Ronoxan A $(0,08 \%)$ & 6,6 & 1,2 \\
\hline \multirow[t]{2}{*}{ “ } & $+\mathrm{PA}(0,01 \%)+$ & & \\
\hline & $\mathrm{BHT}(0,01 \%)$ & 7,3 & 1,4 \\
\hline \multirow[t]{2}{*}{ “ } & $+P A(0,01 \%)+$ & & \\
\hline & $\mathrm{BHA}(0,01 \%)$ & 7,9 & 1,5 \\
\hline \multirow[t]{2}{*}{ “ } & $\begin{array}{l}+\operatorname{PA}(0,01 \%)+ \\
\operatorname{BHA}(0,005 \%)+\end{array}$ & & \\
\hline & BHT $(0,005 \%)$ & 7,8 & 1,4 \\
\hline “ & + BHT (0,02\%) & 8,3 & 1,5 \\
\hline " & $+\mathrm{BHA}(0,02 \%)$ & 9,0 & 1,7 \\
\hline \multirow[t]{2}{*}{ “ } & $+\operatorname{BHT}(0,01 \%)+$ & & \\
\hline & $\mathrm{BHA}(0,01 \%)$ & 9,8 & 1,8 \\
\hline “ & $+\mathrm{GP}(0,02 \%)$ & 14,1 & 2,6 \\
\hline “ & $+\mathrm{TBHQ}(0,01 \%)$ & 23,8 & 4,4 \\
\hline \multirow[t]{2}{*}{ “ } & + ANTRACINE 220 & & \\
\hline & $(0,005 \%)$ & 25,7 & 4,8 \\
\hline “ & $+\operatorname{TBHQ}(0,02 \%)$ & 35,0 & 6,5 \\
\hline
\end{tabular}

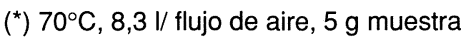

Se observó, confirmando resultados anteriores (Ke, 1977), que TBHQ, cuya concentración máxima permitida en Estados Unidos y en Brasil es de 0,02\%, a la mitad de esa concentración continúa siendo más efectivo que GP, $\mathrm{BHA}$ y $\mathrm{BHT}$ al $0,02 \%$. Se aumenta el periodo de inducción 4,4 veces con TBHQ 0,01\% y 1,7 y 1,5 veces con $\mathrm{BHA}$ y $\mathrm{BHT} 0,02 \%$, respectivamente.

En la concentración máxima ensayada $(0,02 \%)$, los tocoferoles (Tocomix D) no mostraron ningún efecto sobre el aceite, mientras que el palmitato de ascorbilo a $0,02 \%$ y $0,05 \%$ mostró un efecto poco significativo.

Se utilizaron también mezclas de antioxidantes y preparados comerciales conteniendo agentes quelantes y/o sinergistas. En el caso del PA se observó una pequeña mejora en la eficiencia utilizando una mezcla con alfa- 
tocoferol y lecitina (siendo la concentración de PA $0,02 \%$ ), comparada con PA puro al $0,02 \%$.

Las mezclas de PA con BHT y BHA no mejoran la eficiencia de los compuestos butilados utilizados aisladamente y a la misma concentración total de antioxidante.

Con una mezcla de BHA + BHT no se observó un aumento importante del periodo de inducción respecto a los correspondientes cuando se agregaron los compuestos puros a la misma concentración.

En resumen, los resultados obtenidos muestran que para el aceite de hígado de merluza, el TBHQ es más efectivo a la concentración máxima permitida $(0,02 \%)$ y aún a menor concentración $(0,01 \%)$, que los demás antioxidantes ensayados.

\section{AGRADECIMIENTOS}

Al Comité Nacional de Biotecnología (Uruguay), al Laboratorio de Oleos e Gorduras (Unicamp, Brasil) y a INAPE (Instituto Nacional de Pesca, Uruguay), gracias a los cuales este trabajo fue posible.

\section{BIBLIOGRAFIA}

Ackman, R.G. (1969).-«Lipids» en «Methods in Enzimology» Vol. XIV, Cap. 49.-J.L. Lowestein (Ed.).-Academic Press, New York.

AOCS Official Methods and Recomended Practices of the American Oil Chemists Society (1988).-R.C. Walker (Ed.).-3a edición.-Champaing.

De Koning, A. and Milkovitch, S. (1991).-«The synthesis of a number of ethoxyquin analogues and their evaluation as antioxidants in fish oil».Fat Sci. Technol. 93, 378-382.

De Man, J.M. and De Man, L.A. (1984).-«Automated AOM test for fat stability".-J. Am. Oil Chemists'Soc. 61, 534-536.

De Man, J.L.; Tie, F. and De Man, L. (1987).-«Formation of short chain volatile organic acids in the automated AOM method".-J. Am. Oil Chemists'Soc. 64, 993-996.

Digesto Municipal (1990).-Art. D1226.-Libro VI: De la Higiene y Asistencia Social; Intendencia Municipal de Montevideo.

España (1989).-Ministerio de Relaciones con las Cortes y de la Secretaría del Gobierno; Orden 2265 del 25 de enero de 1989.

Gerbshbein, L.L. and Singh, E.J. (1969).-«Hidrocarbons of dogfish and cod livers and herring oil».-J. Am. Oil Chemists'Soc. 46, 554-557.
Han, D.; Yi, O.S. and Shin, H.K. (1991).-«Solubilization of Vitamin C in fish oil and synergistic effect with Vitamin $E$ in retarding oxidation".-J. Am. Oil Chemists'Soc. 68, 740-743.

Hardy, R. and Keay, J.N. (1972)--«Seasonal variations in the chemical composition of cornish mackerel, Scomber scombrus (L), with detailed reference total lipids».-J. Food Tech. 7, 125-137.

Hartman, L. and Lago, R.C.A. (1973).-«Rapid preparation of fatty acid methyl esters from lipids".-Lab. Prat. 22, 475-476.

IUPAC «Standard Methods for the Analysis of Oils Fats and Derivatives» (1987).-C. Paquot and A. Hautfenne (Ed.).-7ª edición.-Blackwell Scientific Publications.

Kaitaranta, J.K. (1992) - "Control of lipid oxidation in fish oil with various antioxidative compounds».-J. Am. Oil Chemists'Soc. 69, 810-813.

Ke, P.J., Nash, D.M. and Ackman, R.G. (1977).-«Mackerel lipids as an unsaturated fat model system for the determination of the antioxidative potency of TBHQ and other antioxidant compounds".-J. Am. Oil Chemists'Soc. 54, 417-420.

Läubli, M.W. and Bruttel, P.A. (1986).-«Determination of the oxidative stability of fats and oils: comparison between the Active Oxigen Method (AOCS Cd 12-57) and the Rancimat Method".-J. Am. Oil Chemists'Soc 63, 792-795.

Lovaas, E. (1991).-«Antioxidative effects of polyamines».-J. Am. Oil Chemists'Soc. 68, 353-358.

Pacheco, M.T. (1991 a).-«Obtençao e fracionamento do óleo do fígado de Tubarao Azul (Prionace glauca) e sua estabilizaçao com antioxidantes".-Tesis de Maestría en Ingeniería de los Alimentos; FEA/UNICAMP, SP, Brasil.

Pacheco, M.T.; Barrera-Arellano, D. and Esteves, W. (1991 b).-«Shark liver oil fractionation and its stabilization with natural and synthetic antioxidants".-Proceedings of the International Meeting on Fats \& Oils Technology.-Symposium and Exhibition, p. 244-247 Campinas, SP, Brasil.

Sherwin, E.R. (1968).-«Methods for Stability and Antioxidant Measurement".-J. Am. Oil Chemists'Soc. 45, 632A-649A.

Shukla, V. and Perkins, E.G. (1991).-«The presence of oxidative polymeric materials in encapsulated fish oils".-Lipids 26, 23-26.

Stansby, M.E. (1981).-«Reliability of fatty acid values purpoting to represent composition of oil from different species of fish".-J. Am. Oil Chemists'Soc. 58, 13-16.

Thorisson, S.; Gunstone, F. and Hardy, R. (1992).-«The antioxidant properties of ethoxyquin and of some of its oxidation products in fish oil and meal».-J. Am. Oil Chemists'Soc. 69, 806-809.

Wada, S. and Fang, X. (1992).-«The synergistic antioxidant effect of Rosemary extract and alpha-tocopherol in sardine oil model system and frozen-crushed fish meat».-J. Food Process. Preserv. 16, 263-274.

(Recibido: Marzo 1993) 